\title{
Assessment of Opicinumab in Acute Optic Neuritis Using Multifocal Visual Evoked Potential
}

\author{
Alexander Klistorner ${ }^{1} \cdot$ Yi Chai $^{2} \cdot$ Letizia Leocani $^{3} \cdot$ Philipp Albrecht $^{4} \cdot$ Orhan Aktas $^{4} \cdot$ Helmut Butzkueven $^{5}$. \\ Tjalf Ziemssen $^{6} \cdot$ Focke Ziemssen $^{7}$. Jette Frederiksen ${ }^{8} \cdot$ Lei Xu $^{2} \cdot$ Diego Cadavid $^{2,9}$ on behalf of RENEW MF-VEP \\ Investigators
}

Published online: 28 September 2018

(c) The Author(s) 2018

\begin{abstract}
Background Multifocal visual evoked potential (MF-VEP) assesses a wider visual field than full-field VEP (FF-VEP) and potentially offers a more precise analysis of optic nerve injury and repair following optic neuritis. MF-VEP may offer advantages over FF-VEP as an endpoint in clinical trials of remyelinating therapies.

Objective MF-VEP testing was used to study changes in visual pathways in 48\% of RENEW [phase II, opicinumab (antiLINGO-1; BIIB033) vs. placebo after first acute unilateral optic neuritis] participants.

Methods This exploratory MF-VEP RENEW substudy compared mean outcomes at weeks 24 and 32 among participants in the intent-to-treat (ITT; $n=39 ; 72 \%$ female; mean age: 32.3 years) and per-protocol (PP; $n=31 ; 71 \%$ female; mean age: 32.2 years) populations in affected and fellow eye latency from fellow eye baseline latency and affected and fellow eye amplitude from their own baselines. Treatment differences were evaluated using analysis of covariance (week 24) and a mixedeffect model of repeated measures (week 32). Last observation carried forward was used to impute missing data at week 24. Results A trend for improvement in affected eye MF-VEP latency with opicinumab versus placebo was seen in the ITT and PP populations at weeks 24 and 32. Both treatment groups in the ITT population experienced partial recovery of amplitude in the affected eye at week 32. Notably, the mean change in fellow eye amplitude at weeks 24 and 32 was -17.57 and -31.41 nanovolts $(\mathrm{nV})$ in placebo but only -0.59 and $1.93 \mathrm{nV}$ in the opicinumab group [differences at weeks 24 and $32: 16.98 \mathrm{nV}$ $(p=0.050)$ and $33.33 \mathrm{nV}(p<0.01)$, respectively].

Conclusion Results from this substudy showed advantages of MF-VEP over FF-VEP in multicenter studies of central nervous system reparative therapies and provide novel evidence that fellow eye visual pathway amplitude loss occurs after optic neuritis but can potentially be prevented by opicinumab treatment.
\end{abstract}

Registration ClinicalTrials.gov identifier NCT01721161.

Drs. Chai, $\mathrm{Xu}$, and Cadavid have since left Biogen. Dr. Cadavid is a full-time employee of Fulcrum Therapeutics.

The members of RENEW MF-VEP Investigators are listed in acknowledgements.

$\triangle$ Diego Cadavid

cadavid.diego@gmail.com

1 Department of Ophthalmology, University of Sydney, Sydney, NSW, Australia

2 Biogen, Cambridge, MA, USA

3 Neurological Department and Experimental Neurophysiology Unit, Institute of Experimental Neurology (INSPE), University Hospital-IRCCS San Raffaele, Milan, Italy

4 Department of Neurology, Medical Faculty, Heinrich-Heine-Universität Düsseldorf, Düsseldorf, Germany
5 Department of Neuroscience, Central Clinical School, Monash University Alfred Campus, Melbourne, VIC, Australia

6 MS Center Dresden, Center of Clinical Neuroscience, University Hospital Carl Gustav Carus, Dresden University of Technology, Dresden, Germany

7 Center for Ophthalmology, Eberhard Karls University of Tübingen, Tübingen, Germany

8 Department of Neurology, Rigshospitalet Glostrup and University of Copenhagen, Copenhagen, Denmark

9 Fulcrum Therapeutics, Cambridge, MA, USA 


\section{Key Points}

The RENEW primary study (NCT01721161), which assessed the efficacy and safety of opicinumab (antiLINGO-1; BIIB033) in participants after a first episode of acute optic neuritis (AON), included a substudy to measure changes in latency and amplitude in both the affected and fellow eyes using multifocal visual evoked potential (MF-VEP).

Latency changes measured by MF-VEP were highly correlated with full-field VEP (FF-VEP) latency changes measured both within this substudy and in the primary RENEW study, confirming the treatment effect demonstrated by opicinumab in the RENEW study.

MF-VEP appears superior to FF-VEP by showing similar treatment effects on latency delay with a smaller sample size and revealing treatment effects on amplitude that were not apparent with FF-VEP.

Treatment with opicinumab prevented the progressive fellow eye visual pathway amplitude loss following AON that was demonstrated by the placebo group and also reduced amplitude loss in the affected eye following AON.

\section{Introduction}

Opicinumab (anti-LINGO-1; BIIB033) is a fully human monoclonal antibody against LINGO-1 [1]. Following testing in preclinical models and phase I studies [1, 2], a phase II clinical development program was initiated to investigate opicinumab as a potential treatment for central nervous system (CNS) demyelinating disorders.

RENEW was the first multicenter randomized clinical trial to investigate a potential CNS remyelinating therapy in humans. Opicinumab was compared with placebo (in addition to standard intravenous [IV] corticosteroid pulse therapy) in participants with a first episode of acute optic neuritis (AON), with treatment starting 3-4 weeks after the first symptom and continuing for up to 8 months. A first AON episode was selected because it is often the first manifestation of multiple sclerosis (MS) and allows the study of two potential mechanisms for LINGO-1 blockade: remyelination [via improved latency of the visual evoked potential (VEP)], and neuroprotection [via preservation of retinal nerve fiber layer thickness measured by spectral domain optical coherence tomography (SD-OCT) and VEP amplitude]. RENEW primary endpoint analysis demonstrated some improvement in full-field (FF)-VEP latency recovery in the affected eye relative to the unaffected eye at baseline, consistent with enhanced remyelination of the recently affected optic nerve [3].

FF-VEP is a sensitive and objective tool for detecting latency abnormalities in the visual pathway [4] and represents combined activity of the central part of the visual field. In contrast, multifocal (MF)-VEP is performed by simultaneously stimulating multiple individual regions of the visual field using different pseudorandom sequences for different segments. By cross-correlating each sequence with the raw electroencephalogram signal, responses to unique sequences corresponding to each of the individually stimulated segments are extracted. Thus, a wider and more detailed assessment of the visual field is performed using MF-VEP, allowing better identification of regional changes in amplitude and latency and potentially a more precise analysis of injury and repair following AON [5-8]. Furthermore, since the VEP signal is generated by dipoles oriented perpendicularly to the brain surface, the upper and lower calcarine visual cortex produces mostly opposing waveforms and, as a result, FF-VEP but not MF-VEP is prone to cancellation [9]. Individual MF-VEP waveforms are generated independently by small areas of the visual cortex and are not affected by signal cancellation between the upper and lower fields. MF-VEP reproducibility over time is excellent $[10,11]$, as is its sensitivity to detect changes [12]. A technical feasibility study of MF-VEP latency demonstrated a variability (over two tests) within $2 \mathrm{~ms}$ for healthy controls and individuals with preexisting optic neuritis and a strong correlation between the first and second tests $(r=0.9997)$ [13].

Prolonged latencies and decreased or absent cortical MFVEP amplitudes with monocular stimulation are hallmarks of AON $[6,14,15]$ and are caused by inflammatory demyelination and conduction block and/or axonal injury. Significant amplitude recovery and some latency recovery typically occur spontaneously via resolution of inflammation, ion channel redistribution in demyelinated axons, and remyelination [16]. In RENEW, we included an MF-VEP substudy in $48 \%$ of participants to measure changes in latency and amplitude in the affected and fellow eye visual pathways in greater detail. This is the first time MF-VEP has been used in a multicenter therapeutic clinical trial.

\section{Methods}

\subsection{RENEW Study Design}

The design and methodology of RENEW (NCT01721161) have been described previously [3]. Briefly, participants aged 18-55 years experiencing a first unilateral AON episode and with no history of MS were randomized 1:1 to placebo or opicinumab $100 \mathrm{mg} / \mathrm{kg}$ IV every 4 weeks from 
baseline to week 20 (six treatments) with follow-up until week 32. Patients with fellow eye VEP latency abnormalities were excluded from RENEW [3]. Prior to randomization, all participants were treated within 28 days of the first AON symptom onset with high-dose IV corticosteroids. An opicinumab $100 \mathrm{mg} / \mathrm{kg}$ dose was selected for this proofof-biology study because it was the highest dose tested in phase I trials, was found to be safe and well-tolerated, and is predicted to result in CNS exposures above the $90 \%$ effective concentration for remyelination in rodent pharmacology models [2]. All participants provided written informed consent, the study was performed in accordance with the International Conference on Harmonisation Good Clinical Practice guidelines and the Declaration of Helsinki $[17,18]$, and all investigators obtained approval from their local ethics committees.

\subsection{Multifocal Visual Evoked Potential (MF-VEP) Methodology}

As specialist equipment was provided to the sites that participated in the MF-VEP substudy, it was not possible to perform MF-VEP at all locations. Thirteen of the sites participating in RENEW were selected for the MF-VEP substudy and supplied with MF-VEP machines (VisionSearch 1 system; VisionSearch, Sydney, NSW, Australia) by the sponsor (Biogen). Technicians at all 13 sites were trained in person and were qualified to perform MF-VEP by one of the authors (AK), followed by formal assessment by the central reader (Duke University Clinical Research Center, Durham, NC, USA). MF-VEP measurements were taken at screening, baseline, weeks 4, 8, 12, 16, 20, and 24 ( \pm 3 days), and at week 32 ( \pm 7 days). For participants who discontinued study treatment early or withdrew prematurely from the study, MF-VEP was measured at the early termination visit that was completed 4 weeks ( \pm 7 days) after their last study treatment. The individual segments assessed by MF-VEP are shown in Fig. 1a.

Fifty-six cortically scaled segments containing a $4 \times 4$ grid of black-and-white checks reversing according to pseudorandom sequences were used for monocular visual field stimulation (Fig. 1b). The visual stimulus was generated on a 21-inch high-resolution liquid crystal display screen and occupied $48^{\circ}$ of the visual field. All participants were optimally refracted for near vision and the pupils were not dilated. They were seated $30 \mathrm{~cm}$ from the screen. Four gold-cup electrodes (Grass Technologies, West Warwick, RI, USA) were used for bipolar recording: two electrodes positioned $4 \mathrm{~cm}$ on either side of the inion, one electrode in the midline $2.5 \mathrm{~cm}$ above the inion, and one electrode $4.5 \mathrm{~cm}$ below the inion. Conductive gel was injected under each electrode $(0.5 \mathrm{~mL})$. Electrical signals were recorded along two channels: vertical (using superior and inferior

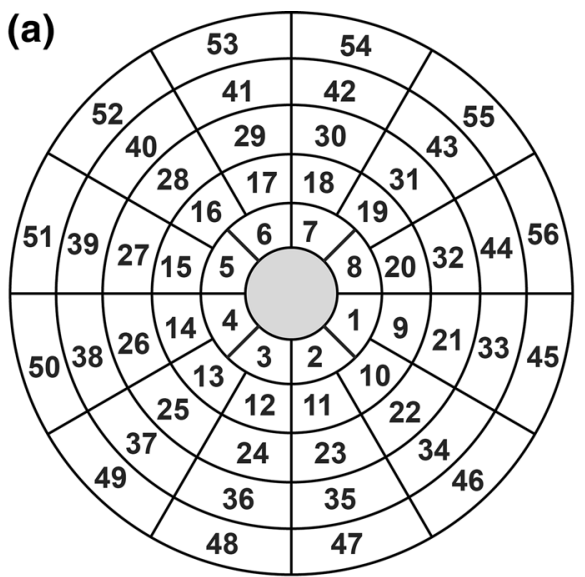

(b)

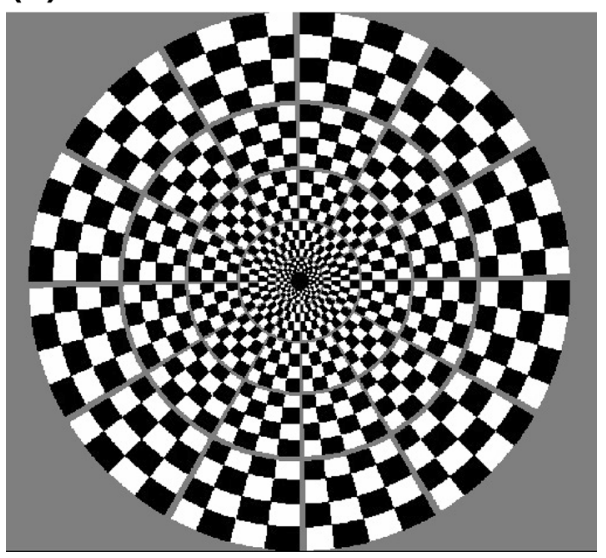

Fig. 1 Individual segments assessed using a multifocal visual evoked potential and $\mathbf{b}$ reversing black-and-white checks used for monocular stimulation

electrodes) and horizontal (using left and right electrodes). A ground electrode was placed on an ear lobe. Each of the 56 segments was stimulated with a different frequency and the resultant VEP responses were amplified 100,000 times and band-pass filtered (1-20 Hz). For amplitude analysis, the largest (between two channels) peak-trough amplitude within the interval of 70-200 ms was determined. The second peak of the maximum amplitude wave for each visual field segment was used for latency analysis. The software of the MF-VEP equipment calculated the average amplitude and frequency from the individual segments. If a segment had no detectable amplitude, then a reading of 0 nanovolts $(\mathrm{nV})$ was assigned. Latency measurements were only assigned to segments with amplitude readings not equal to $0 \mathrm{nV}$. Segments with undetectable amplitude readings did not have a latency assigned. No compensation for peripheral sectors and no imputations were performed.

The blinded central reader was responsible for MF-VEP data quality control and for latency and amplitude measurement at the individual subject level using progression 
analysis for each of the 56 segments from all available study visits.

\subsection{Full-Field Visual Evoked Potential (FF-VEP) and Spectral Domain Optical Coherence Tomography (SD-OCT) Methodology}

FF-VEP latency and amplitude as well as retinal ganglion cell layer/inner plexiform layer (RGCL/IPL) thickness, measured by SD-OCT, were assessed in the main RENEW study. Both methods used a standard protocol provided by the central reading center to ensure reproducibility and consistency, as described previously [3]. The FF-VEP protocol was developed to comply with guidelines from both the International Society for Clinical Electrophysiology of Vision and the American Clinical Neurophysiology Society. SD-OCT scan images were obtained using a Spectralis ${ }^{\circledR}$ (Heidelberg Engineering, Heidelberg, Germany) or Cirrus ${ }^{\mathrm{TM}}$ (Carl Zeiss Meditec, Dublin, CA, USA) system. Central reading, certification, and quality control procedures, as well as the optical coherence tomography (OCT)-scanning protocol, complied with the APOSTEL (Advised Protocol for OCT Study Terminology and Elements) recommendations $[3,19]$. The central reader evaluated all data and provided the final values and interpretations. FF-VEP and RGCL/IPL data for substudy participants were obtained from the main study results.

\subsection{Statistical Analyses}

In this substudy, the primary efficacy analysis was change in MF-VEP-affected eye latency at week 24 (end of treatment) from baseline of the unaffected (fellow) eye in milliseconds. A key hurdle when measuring changes from baseline in AON VEP latency is that pretreatment latency delay from demyelination cannot be determined because of the high prevalence of conduction block and residual acute inflammation in the affected eye optic nerve. To overcome this limitation, RENEW required each participant to have a normal fellow (unaffected) eye to acquire the normal, preAON onset latency values.

In addition to latency, exploratory efficacy analyses were included to measure changes from baseline in affected and fellow eye MF-VEP amplitude over time expressed in nanovolts. Segments without recordable affected eye amplitude were scored as $0 \mathrm{nV}$. To visualize changes in amplitude from baseline in all 56 MF-VEP segments in the affected and fellow eyes, heat map displays were generated for each visit ( $R$ statistical software), with green representing amplitude gains (improvements) from baseline and red representing amplitude losses (worsening) from the baseline value of the respective eye.
All participants in the MF-VEP substudy were included in the intent-to-treat (ITT) population and received at least one dose of study drug or placebo. MF-VEP substudy participants who were included in the per-protocol (PP) population in the full trial (defined as those who completed the study, did not miss more than one treatment dose, and did not receive MS disease-modifying therapy) formed the PP population in the MF-VEP substudy. The PP population analysis was post hoc in the MF-VEP substudy.

Demographic data were summarized using descriptive statistics. Values for affected and unaffected fellow eye MFVEP latency and amplitude at each visit were summarized using descriptive statistics by treatment group. Treatment differences in MF-VEP latency and amplitude were evaluated using analysis of covariance (ANCOVA) at week 24 and a mixed-effect model of repeated measures (MMRM) through week 32. Last observation carried forward was used to impute missing data at week 24 in the ANCOVA evaluation. ANCOVA and MMRM models were adjusted for the baseline value of the outcome being examined.

Participants with FF-VEP latency recovery at week 24 were prespecified as those with an affected eye FF-VEP latency $\leq 10 \%$ worse than the fellow eye. Mean differences in MF-VEP latency and amplitude at week 24 in participants with and without FF-VEP latency recovery from baseline impairment were evaluated by ANCOVA. Correlations of MF-VEP with FF-VEP and RGCL/IPL thickness were assessed using pairwise correlation and the Pearson correlation coefficient.

The MF-VEP substudy was not designed to reach significance for testing treatment effect on latency or amplitude. $p$ values reported were not adjusted for multiple testing. The magnitude of the treatment effect was unknown at the time the substudy was designed because RENEW was the first time the efficacy of opicinumab for CNS remyelination was examined in the clinic. Data were checked for normality and were found to be normally distributed.

\section{Results}

\subsection{Enrollment in the MF-VEP Substudy and Baseline Characteristics}

Thirty-nine (48\%) participants in the RENEW ITT population were included in the MF-VEP substudy, mandatory at all 13 sites selected for substudy participation. Of the 39 participants, 21 were randomized to opicinumab and 18 to placebo. The two treatment groups were similar in baseline demographics except for conduction block in the affected eye, which was more frequent in the opicinumab group (Table 1). Fellow eye MF-VEP latency and amplitude values were normal at baseline; average latency for a 
Table 1 Baseline demographic characteristics of all participants in the MF-VEP substudy

\begin{tabular}{llll}
\hline Characteristic & Placebo $(n=18)$ & Opicinumab $(n=21)$ & All participants $(N=39)$ \\
\hline Female (\%) & 78 & 67 & 72 \\
White (\%) & 94 & 100 & 97 \\
Mean \pm SD age (years) & $31.8 \pm 9.93$ & $32.7 \pm 7.90$ & $32.3 \pm 8.78$ \\
Weight (kg) [median (range)] & $75.0(47-119)$ & $72.2(57-106)$ & $75.0(47-119)$ \\
Height (cm) [median (range)] & $170.0(155-194)$ & $171.0(158-185)^{\mathrm{a}}$ & $170.0(155-194)^{\mathrm{b}}$ \\
Mean \pm SD MF-VEP latency of the fellow eye (ms) & $144.45 \pm 6.22$ & $147.68 \pm 5.33^{\mathrm{a}}$ & $146.15 \pm 5.92^{\mathrm{b}}$ \\
Mean \pm SD MF-VEP latency of the affected eye (ms) & $167.61 \pm 19.90$ & $166.65 \pm 19.69^{\mathrm{a}}$ & $167.10 \pm 19.53^{\mathrm{b}}$ \\
Mean \pm SD MF-VEP amplitude of the fellow eye (nV) & $156.77 \pm 57.30$ & $167.36 \pm 34.60^{\mathrm{a}}$ & $162.34 \pm 46.39^{\mathrm{b}}$ \\
Mean \pm SD MF-VEP amplitude of the affected eye (nV) & $87.21 \pm 48.57$ & $78.36 \pm 57.63^{\mathrm{a}}$ & $82.56 \pm 53.01^{\mathrm{b}}$ \\
No. $(\%)$ with $<60 \%$ measurable segments & $4(22)$ & $9(45)^{\mathrm{a}}$ & $13(34)^{\mathrm{b}}$
\end{tabular}

$M F$-VEP multifocal visual evoked potential, $n V$ nanovolts, $S D$ standard deviation

${ }^{\mathrm{a}} n=20$; baseline height and MF-VEP latency and amplitude not available for one participant

${ }^{\mathrm{b}} n=38$; baseline height and MF-VEP latency and amplitude not available for one participant

${ }^{c}$ Participants with recordable latency in $<60 \%$ of segments at baseline were considered to have conduction block at baseline

control cohort was previously reported as $145.9 \pm 4.6 \mathrm{~ms}$ and $149.3 \pm 5.1 \mathrm{~ms}[13,20]$ for both sides and the normal average range for amplitude was reported as $156-210 \mathrm{nV}$ [21]. The mean affected eye baseline amplitude was reduced by approximately half in both treatment groups compared with the fellow eye (Table 1).

Thirty-one (45\%) of 69 participants in the main study PP population were represented in the MF-VEP substudy PP population, with 15 randomized to opicinumab and 16 to placebo (Table 2). The substudy population was representative of the overall participant population in RENEW.

\subsection{Recovery of MF-VEP Latency in the Affected Eye}

Both groups showed MF-VEP latency prolongation at the end of treatment (week 24) and end of study (week 32). In the ITT population, the adjusted mean change in latency in the opicinumab group compared with placebo at week 24 was $-4.97 \mathrm{~ms}$ [ $95 \%$ confidence interval $(\mathrm{CI})-16.03$ to $6.09 ; p=0.37]$; in the PP population, it was $-11.78 \mathrm{~ms}(95 \%$ CI -24.28 to 0.73 ; $p=0.06$; by ANCOVA; Fig. 2 ). The corresponding data at Week 32 were $-3.82 \mathrm{~ms}(95 \% \mathrm{CI}-15.10$ to $7.46 ; p=0.50)$ and $-9.38 \mathrm{~ms}(95 \% \mathrm{CI}-22.22$ to 3.46 ; $p=0.15$; by MMRM; Fig. 2). Although the average improvement in MF-VEP latency was numerically almost twofold higher with opicinumab than with placebo at both timepoints in the PP population, the data were highly variable and the

Table 2 Baseline demographic characteristics of participants in the MF-VEP substudy per-protocol population

\begin{tabular}{llll}
\hline Characteristic & Placebo $(n=16)$ & Opicinumab $(n=15)$ & All participants $(N=31)$ \\
\hline Female $(\%)$ & 75 & 67 & 71 \\
White $(\%)$ & 94 & 100 & 97 \\
Mean \pm SD age (years) & $32.8 \pm 10.14$ & $31.7 \pm 7.83$ & $32.2 \pm 8.97$ \\
Weight (kg) [median (range)] & $75.0(51-119)$ & $75.0(57-106)$ & $75.0(51-119)$ \\
Height (cm) [median (range)] & $170.0(157-194)$ & $172.5(159-182)^{\mathrm{a}}$ & $170.5(157-194)^{\mathrm{b}}$ \\
Mean \pm SD MF-VEP latency of the fellow eye (ms) & $143.67 \pm 6.01$ & $147.66 \pm 4.98^{\mathrm{a}}$ & $145.53 \pm 5.82^{\mathrm{b}}$ \\
Mean \pm SD MF-VEP latency of the affected eye (ms) & $169.80 \pm 19.80$ & $162.87 \pm 18.37^{\mathrm{a}}$ & $166.56 \pm 19.14^{\mathrm{b}}$ \\
Mean \pm SD MF-VEP amplitude of the fellow eye (nV) & $159.23 \pm 56.30$ & $163.54 \pm 38.40^{\mathrm{a}}$ & $161.24 \pm 48.01^{\mathrm{b}}$ \\
Mean \pm SD MF-VEP amplitude of the affected eye (nV) & $83.27 \pm 48.04$ & $82.93 \pm 56.13^{\mathrm{a}}$ & $83.11 \pm 51.05^{\mathrm{b}}$ \\
No. $(\%)$ with $<60 \%$ measurable segments & $6(25)$ & $6(43)^{\mathrm{a}}$ & $10(33)^{\mathrm{b}}$ \\
\hline
\end{tabular}

$M F$-VEP multifocal visual evoked potential, $n V$ nanovolts, $S D$ standard deviation

${ }^{\mathrm{a}} n=14$; baseline height and MF-VEP latency and amplitude not available for one participant

${ }^{\mathrm{b}} n=30$; baseline height and MF-VEP latency and amplitude not available for one participant

${ }^{c}$ Participants with recordable latency in $<60 \%$ of segments at baseline were considered to have conduction block at baseline 

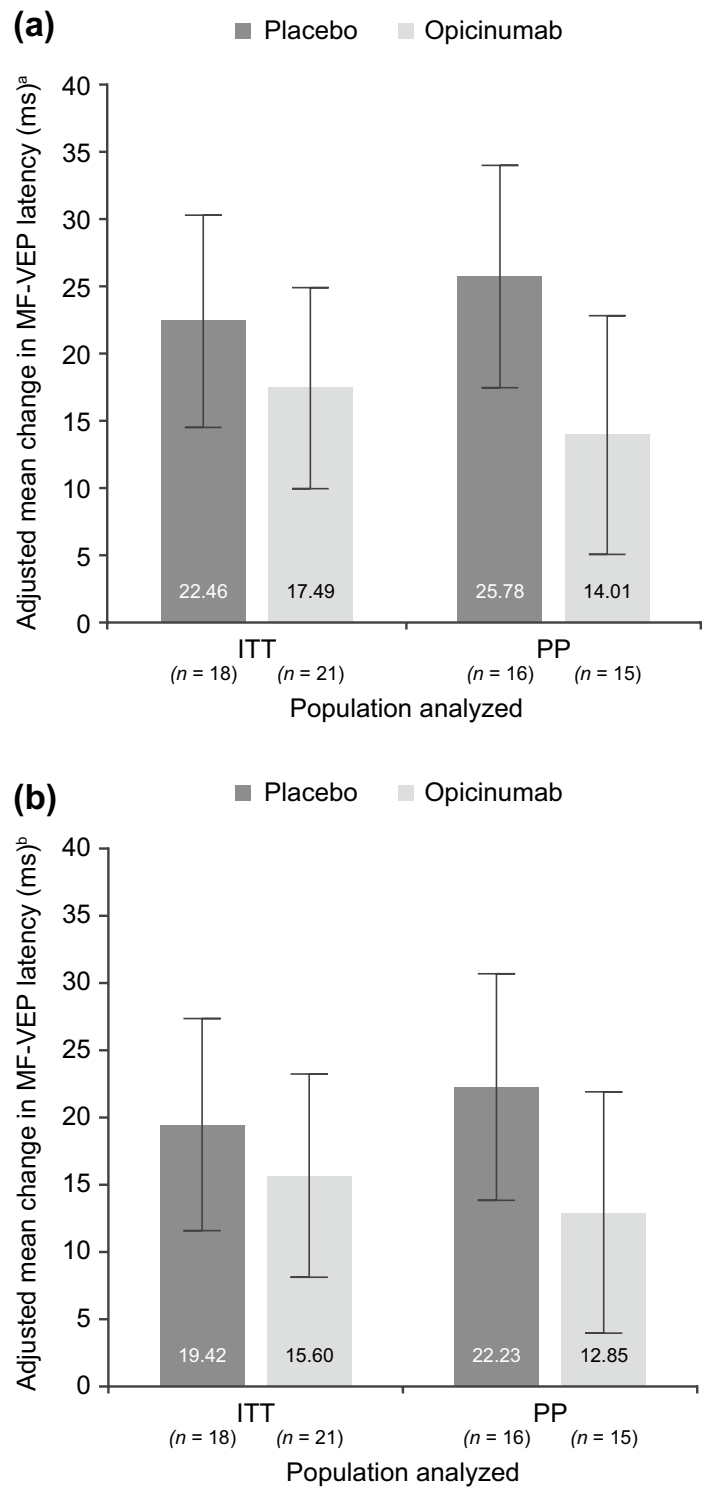

Fig. 2 Mean change in MF-VEP latency, adjusted for the baseline latency of unaffected fellow eye, at a week 24 and b week 32 in the affected eye compared with the unaffected fellow eye at baseline in the substudy ITT and PP populations. Error bars represent 95\% CIs. ${ }^{\mathrm{a}}$ Analyzed by analysis of covariance. ${ }^{\mathrm{b}}$ Analyzed by mixed-effect model of repeated measures. $C I$ confidence interval, ITT intent-totreat, $M F-V E P$ multifocal visual evoked potential, $P P$ per protocol

study was therefore underpowered to detect a statistically significant treatment effect.

A post hoc comparison of estimated effect size for change in MF-VEP and FF-VEP latency for opicinumab versus placebo at week 24 in the ITT population showed that MF-VEP demonstrated a larger treatment effect size than FF-VEP (Table 3).

\subsection{Recovery of MF-VEP Amplitude in the Affected Eye}

Both treatment groups experienced amplitude recovery in the affected eye from baseline; there was no significant between-group difference in recovery at week 24 . However, there was a strong trend for improvement with opicinumab over 32 weeks; the mean gain versus placebo was $22.32 \mathrm{nV}$ (95\% CI -1.26 to $45.89 ; p=0.06$; Fig. 3a; MMRM; ITT population). The recovery in the placebo group peaked by week 16 and appeared to worsen towards the end of the study, whereas the opicinumab group continued to improve through the last study assessment (week 32). The heat map display revealed that the greatest recovery occurred in the segments with recording electrodes closest to the visual cortical area generating the signal (i.e., the upper calcarine visual cortex; Fig. 3a), a consequence of the anatomy of the visual cortex $[9,22]$.

\subsection{MF-VEP Latency and Amplitude in the Fellow Eye}

No change from baseline in fellow eye visual pathway latency was observed in either treatment group over 32 weeks; in the ITT population, the adjusted mean change from baseline at week 24 by MMRM was $0.61 \mathrm{~ms}$ for opicinumab and $2.26 \mathrm{~ms}$ for placebo $(p=0.15)$. The corresponding values at week 32 were $1.92 \mathrm{~ms}$ for placebo and $1.32 \mathrm{~ms}$ for opicinumab $(p=0.52)$.

By contrast, there was a significant, gradual loss of MFVEP amplitude in the placebo group that was not seen in the opicinumab group; in the ITT population at week 24 , the adjusted mean change in the fellow eye MF-VEP amplitude by MMRM was $-17.57 \mathrm{nV}$ in the placebo group but only $-0.59 \mathrm{nV}$ in the opicinumab group [difference in adjusted mean change: $16.98 \mathrm{nV}$ (95\% CI -0.23 to 34.18$) ; p=0.05]$. The corresponding values at week 32 were -31.41 and $1.93 \mathrm{nV}$ in the placebo and opicinumab groups, respectively $(p<0.01$; Fig. 3b). The heat map display of the individual segments showed that the progressive fellow eye visual pathway amplitude loss involved the majority of segments and was greatest in the segments corresponding to the visual cortical neurons closer to the MF-VEP scalp electrodes, similar to the findings in the affected eye visual pathway. In the opicinumab group, only a few segments experienced transient and mild loss of amplitude.

\subsection{Correlations of MF-VEP with FF-VEP and SD-OCT}

A prespecified responder analysis of the RENEW primary endpoint classified responders as those whose affected eye latency at week 24 was within $10 \%$ of the fellow eye latency at baseline [3]. We investigated the relationship between this 
Table 3 Comparison of multifocal visual evoked potential and full-field visual evoked potential effect sizes in the intent-to-treat population of RENEW at week 24

\begin{tabular}{llll}
\hline Outcome & $\begin{array}{l}\text { Estimate (difference in adjusted mean estimated } \\
\text { from model) }\end{array}$ & $\begin{array}{l}\text { SD of change at Week 24 in } \\
\text { placebo }\end{array}$ & $\begin{array}{l}\text { Effect size } \\
(\text { estimate/ } \\
\text { SD) }\end{array}$ \\
\hline $\begin{array}{l}\text { Change in MF-VEP latency at week } 24 \\
\text { for opicinumab vs. placebo }\end{array}$ & 4.97 & 16.729 & 0.297 \\
$\begin{array}{l}\text { Change in FF-VEP latency at week 24 } \\
\text { for opicinumab vs. placebo [3] }\end{array}$ & 3.48 & 15.898 & 0.219 \\
\hline
\end{tabular}

$F F$-VEP full-field visual evoked potential, $M F-V E P$ multifocal visual evoked potential, $S D$ standard deviation

${ }^{a}$ Absolute values are shown

${ }^{\mathrm{b}}$ Estimated with Cohen's $d$, assuming equal variance

responder analysis and the changes observed in latency and amplitude by MF-VEP. The results showed that MF-VEP latency recovery at week 24 was significantly greater in participants who were FF-VEP latency responders at week 24 ( $p<0.01$; Fig. 4). MF-VEP amplitude loss also appeared to be lower in FF-VEP latency responders at week 24 ( $p=0.10$; Fig. 4). In fact, in the affected eye visual pathway, changes in MF-VEP latency at week 24 showed a high correlation with changes in FF-VEP latency in both treatment groups (Table 4). By contrast, the correlation was weaker between MF-VEP and FF-VEP in amplitude changes over 24 weeks and between MF-VEP latency and amplitude with RGCL/ IPL as measured by SD-OCT. OCT was used as a secondary endpoint in RENEW to investigate the potential for opicinumab to provide neuroprotection of RGC following AON (Table 4).

\section{Discussion}

This is the first time MF-VEP has been used in a multicenter therapeutic clinical trial. Results from this exploratory MF-VEP substudy of RENEW showed the feasibility of performing MF-VEP in multicenter studies of candidate CNS reparative therapies. Owing to its low sample size, this exploratory MF-VEP substudy was not designed for statistical significance, but to provide valuable information on the latency and amplitude changes of both the affected and fellow eyes following $\mathrm{AON}$ and the potential treatment effects of opicinumab for both remyelination and neuroprotection in the visual pathway. Importantly, the changes in affected eye latency from baseline of the fellow eye were consistent between the MF-VEP substudy and FF-VEP, the primary endpoint in RENEW [3]. There was also very high concordance in the measurements of affected eye visual pathway latency delay following AON between FF-VEP (measured manually on the first wave by a central reader) and MFVEP (measured automatically on the second wave using a machine algorithm) in both the placebo and opicinumab groups (Table 4). The assessment of treatment effect statistical significance was similar, even though the MF-VEP substudy involved half the number of participants as RENEW.

MF-VEP was hypothesized to be superior to FF-VEP for several reasons, including the ability to detect small outer field defects not detected by FF-VEP, higher sensitivity to assess severely reduced activity, and higher reliability for latency, and especially for amplitude in longitudinal measurements [13]. Predictably, the MF-VEP latency data generated were consistent with the FF-VEP latency results from the overall study (Table 5). Furthermore, participants whose FF-VEP latency recovered to normal/close to normal in RENEW (FF-VEP latency responders; Table 5) showed significantly less prolongation in MF-VEP latency and lower MF-VEP amplitude loss from baseline to week 24 than participants without FF-VEP latency recovery. This confirms the treatment effect observed with FF-VEP in RENEW using separate measurements of latency recovery and highlights the potential of MF-VEP to provide similar information with a smaller sample size in therapeutic trials (39 participants in the MF-VEP substudy vs. 82 participants in RENEW yielded similar statistical differences by treatment; Table 5) [3]. A post hoc analysis of treatment effect size for opicinumab over placebo on latency delay showed that MF-VEP had a larger effect size than FF-VEP, indicating that a potentially smaller sample size is needed with MF-VEP to achieve the same power needed with FF-VEP. While the current study was underpowered to detect a treatment effect, these results suggest that MF-VEP could be a preferred endpoint to FF-VEP in future optic neuritis trials because it could potentially demonstrate similar treatment effects with about half the sample size, in addition to detecting treatment effects on amplitude that are not measurable with FF-VEP.

A novel finding from this exploratory MF-VEP study was that fellow eye visual pathway amplitude loss occurred following unilateral optic neuritis in the opposite eye. Furthermore, this did not occur in the opicinumab group. 
(a)

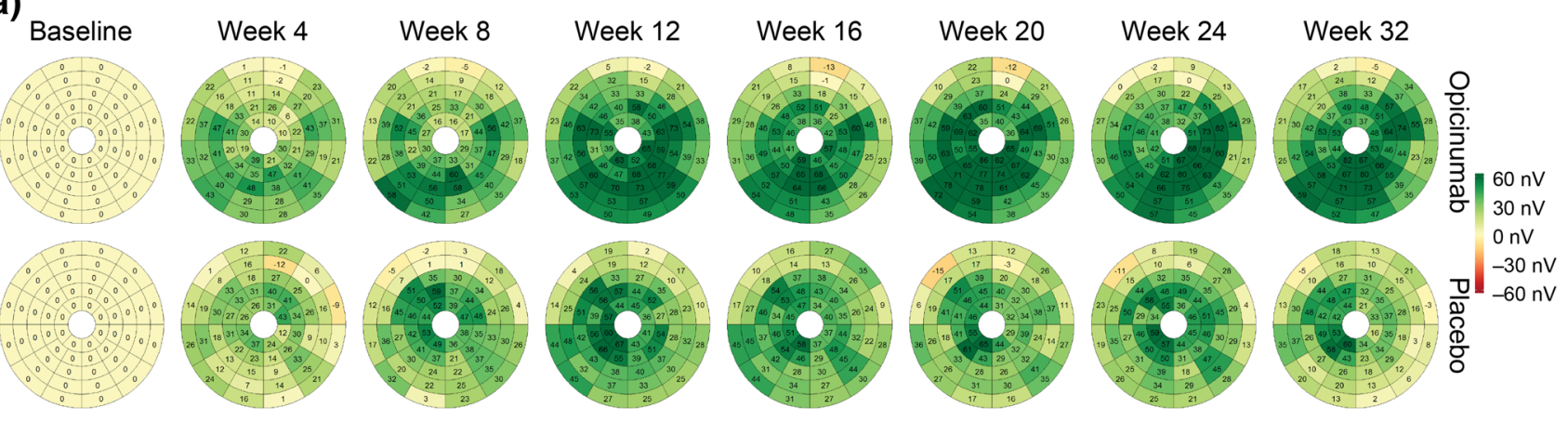

\begin{tabular}{|l|c|c|c|c|c|}
\hline \multirow{2}{*}{ Affected eye $^{a}$} & Average amplitude, $n$; 56 segments & \multicolumn{3}{|c|}{ Difference versus placebo } \\
\cline { 2 - 7 } & Placebo & Opicinumab & Estimated difference & $95 \%$ Cl & $p$ value \\
\hline Change at Week 4 & 23.55 & 25.21 & 1.66 & -18.46 to 21.79 & 0.87 \\
\hline Change at Week 8 & 30.74 & 30.34 & -0.41 & -21.08 to 20.27 & 0.97 \\
\hline Change at Week 12 & 29.92 & 41.56 & 11.64 & -13.26 to 36.53 & 0.35 \\
\hline Change at Week 16 & 34.44 & 43.12 & 8.68 & -15.23 to 32.58 & 0.47 \\
\hline Change at Week 20 & 29.55 & 50.18 & 20.63 & -3.62 to 44.88 & 0.09 \\
\hline Change at Week 24 & 29.86 & 43.52 & 13.65 & -11.66 to 38.96 & 0.28 \\
\hline Change at Week 32 & 25.88 & 48.20 & 22.32 & -1.26 to 45.89 & 0.06 \\
\hline
\end{tabular}

(b)

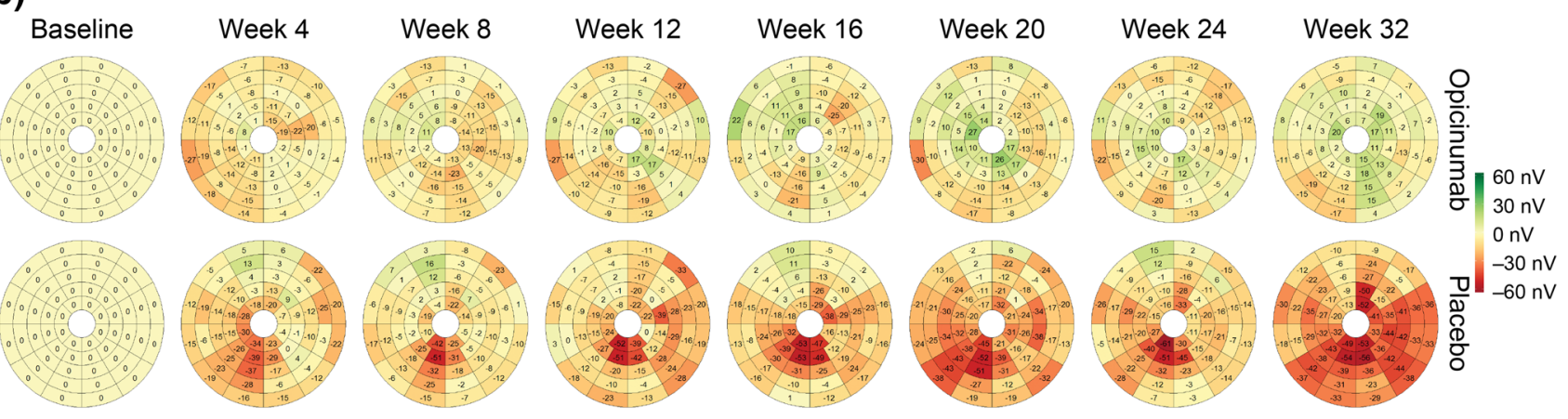

\begin{tabular}{|l|c|c|c|c|c|}
\hline \multirow{2}{*}{ Fellow eye } & \multicolumn{2}{|c|}{ Average amplitude, $\mathrm{nV}$; 56 segments } & \multicolumn{3}{|c|}{ Difference versus placebo } \\
\cline { 2 - 6 } & Placebo & Opicinumab & Estimated difference & $95 \%$ CI & $p$ value \\
\hline Change at Week 4 & -14.02 & -6.13 & 7.89 & -5.93 to 21.71 & 0.25 \\
\hline Change at Week 8 & -12.36 & -4.81 & 7.55 & -9.40 to 24.49 & 0.37 \\
\hline Change at Week 12 & -18.45 & -2.63 & 15.82 & -0.61 to 32.26 & 0.06 \\
\hline Change at Week 16 & -17.52 & -2.85 & 14.68 & -1.46 to 30.81 & 0.07 \\
\hline Change at Week 20 & -24.34 & 1.12 & 25.46 & 8.15 to 42.76 & $<0.01$ \\
\hline Change at Week 24 & -17.57 & -0.59 & 16.98 & -0.23 to 34.18 & 0.05 \\
\hline Change at Week 32 & -31.41 & 1.93 & 33.33 & 16.41 to 50.26 & $<0.01$ \\
\hline
\end{tabular}

Fig. 3 Mean change in multifocal visual evoked potential amplitude by treatment from baseline over 32 weeks in the a affected eye and $\mathbf{b}$ unaffected fellow eye in the intent-to-treat population. In the heat map displays, green represents amplitude gains (improvement) and red represents amplitude losses (worsening) from baseline. Results for the per protocol population were not calculated. ${ }^{\mathrm{a}}$ Compared with baseline value of the affected eye. ${ }^{\mathrm{b}}$ Compared with baseline value of the fellow eye. $C I$ confidence interval, $n V$ nanovolts 

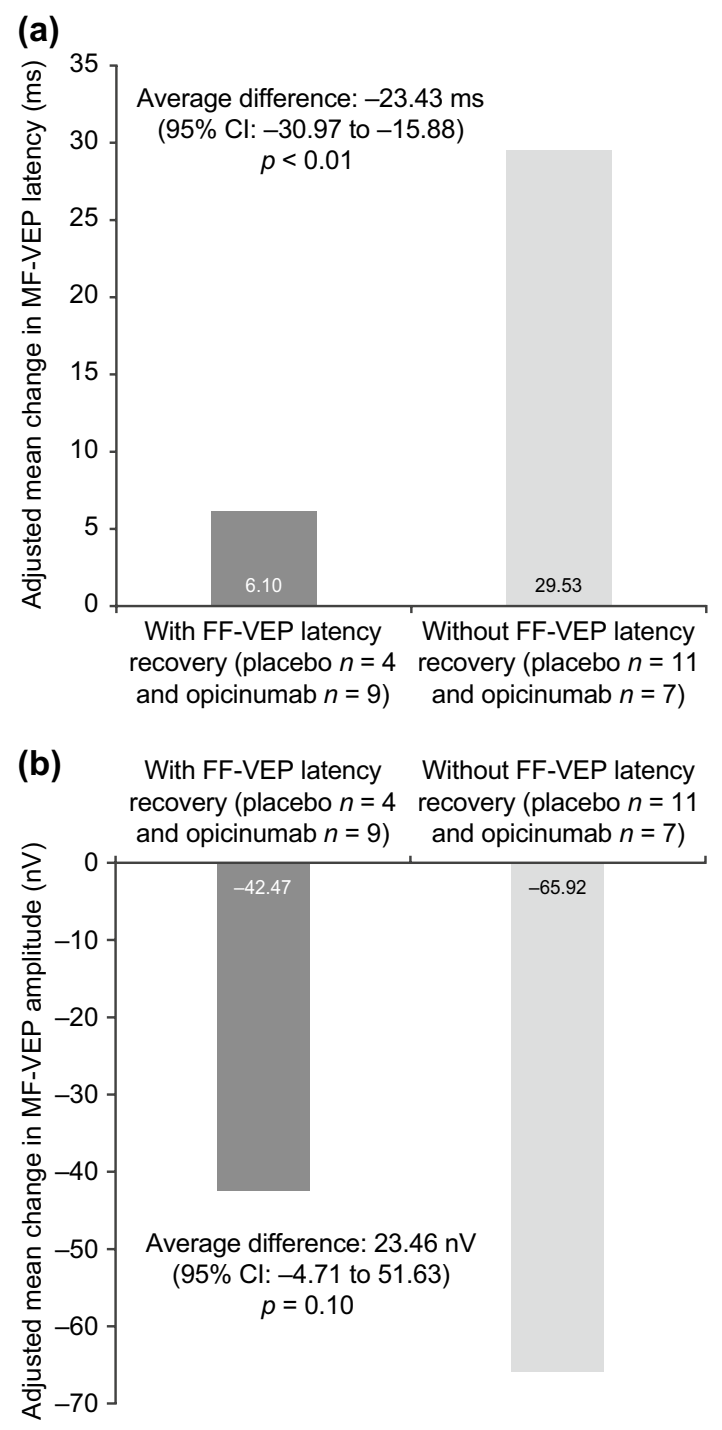

Fig. 4 Adjusted mean differences in $\mathbf{a}$ MF-VEP latency and $\mathbf{b}$ amplitude at week 24 in participants classified with latency recovery using the primary endpoint measure of FF-VEP. FF-VEP latency recovery was defined as affected eye FF-VEP latency at week $24 \leq 10 \%$ worse than the baseline fellow eye; FF-VEP latency was the primary endpoint in RENEW. $C I$ confidence interval, $F F$ - $V E P$ full-field visual evoked potential, $M F-V E P$ multifocal visual evoked potential

Replication and mechanistic investigations to substantiate these novel findings will be important.

There are several potential explanations for the observed fellow eye visual pathway amplitude loss in the placebo arm. First, it may be due to inflammation in the affected eye also affecting the optic chiasm and fellow eye, with edema causing transient conduction block, which could reduce the amplitude. However, the MF-VEP amplitude loss was not temporarily linked to AON onset but, instead, gradually worsened over time following $\mathrm{AON}$ and peaked at 32 weeks. This is the opposite of what would be expected if acute inflammation related to the affected eye AON lesion was the cause.

Second, it may be due to new lesions developing in the fellow eye visual pathway, possibly occurring in the optic nerve, tract or radiation, and/or visual cortex, causing axonal transection and/or axonal demyelination and reduced amplitude. However, the magnetic resonance imaging (MRI) scans at 32 weeks showed very few new cerebral lesions, and the effect would have had to occur in almost all participants in the MF-VEP substudy. As described in previous studies, the T2 sequences used in the RENEW study were appropriate to detect the onset of new lesions in the brain, including the optic radiations [23]. Also, little increase in brain T2 lesion volume was seen in RENEW between baseline and week 32, with no difference between treatment groups, suggesting that new lesion development is an unlikely reason for the new onset of MF-VEP fellow eye visual pathway amplitude loss [3]. A location for these potential new lesions in the optic tract is also possible but unlikely, as optic tract lesions occur infrequently in MS [24-26]. Visual cortex lesions are also possible, but they tend to occur in later phases of MS rather than in this early population $[6,27]$ and are unlikely to be so widespread as to involve the majority of visual fields in most individuals (Fig. 3). If they did occur, these cortical lesions would not be visible by conventional MRI. Finally, a therapeutic effect on new lesion development produced by opicinumab is unlikely, as this antibody had no such effect in animal studies $[28,29]$ or in phase 1 or the RENEW studies.

Third, the amplitude loss could have resulted from anterograde transsynaptic visual cortex degeneration following AON, which is expected to also affect secondary axons coming from the fellow eye visual pathway. MRI studies in various eye diseases have shown secondary structural changes in the visual cortex following unilateral injury to the retina. In the case of anterograde transsynaptic degeneration, it has been hypothesized that axonal breakdown at the site of injury on one side spreads to connected neurons from the contralateral side at the occipital cortex level [30]. There is some evidence of both anterograde and retrograde transsynaptic degeneration in MS. In particular, anterograde transsynaptic degeneration has been observed following optic neuritis and resulted in localized cortical atrophy [31]. Retrograde transsynaptic degeneration, where damage spreads from the visual cortex proximally toward the eye, has also been suggested as a mechanism of axonal damage in MS. Thus, transsynaptic visual pathway degeneration could cause secondary visual pathway dysfunction and may represent a novel therapeutic target to protect the overall integrity of the visual network following unilateral visual pathway lesions, such as in AON [28]. Since LINGO-1 blockade is known to have both remyelinating and neuroaxonal protective effects in various preclinical models $[1,28,32-36]$, it is possible that the observed preservation of MF-VEP amplitude in the 
Table 4 Correlations of the change at week 24 comparing multifocal visual evoked potential, full-field visual evoked potential, and spectral domain optical coherence tomography by treatment group

\begin{tabular}{|c|c|c|c|c|c|c|}
\hline & \multicolumn{3}{|c|}{ Substudy and ITT $(r)$} & \multicolumn{3}{|c|}{ Substudy and PP $(r)$} \\
\hline & Placebo $(n=18)$ & $\begin{array}{l}\text { Opici- } \\
\text { numab } \\
(n=21)\end{array}$ & Total $(N=39)$ & Placebo $(n=16)$ & $\begin{array}{l}\text { Opici- } \\
\text { numab } \\
(n=15)\end{array}$ & Total $(N=31)$ \\
\hline MF-VEP latency and FF-VEP latency & 0.98 & 0.91 & 0.95 & 0.98 & 0.93 & 0.96 \\
\hline MF-VEP amplitude and FF-VEP amplitude & 0.63 & -0.24 & 0.05 & 0.63 & -0.12 & 0.15 \\
\hline MF-VEP latency and MF-VEP amplitude & -0.54 & -0.31 & -0.43 & -0.54 & -0.30 & -0.45 \\
\hline MF-VEP amplitude and FF-VEP latency & -0.42 & -0.58 & -0.48 & -0.42 & -0.52 & -0.45 \\
\hline MF-VEP latency and RGCL/IPL thickness & -0.35 & -0.50 & -0.37 & -0.35 & -0.50 & -0.37 \\
\hline MF-VEP amplitude and RGCL/IPL thickness & 0.28 & 0.51 & 0.39 & 0.28 & 0.51 & 0.38 \\
\hline
\end{tabular}

Pearson correlation analyses were used. All correlation analyses for the affected eye were assessed using baseline of the fellow eye

$F F-V E P$ full-field visual evoked potential, ITT intent to treat, $M F-V E P$ multifocal visual evoked potential, $P P$ per protocol, $R G C L / I P L$ retinal ganglion cell layer/inner plexiform layer

Table 5 Multifocal visual evoked potential substudy and RENEW study outcomes

\begin{tabular}{lll}
\hline Study/outcomes & ITT & PP \\
\hline MF-VEP substudy & & $-11.78 \mathrm{~ms}(p=0.06)$ \\
Change in MF-VEP latency at week 24 for opicinumab vs. placebo & $-4.97 \mathrm{~ms}(p=0.37)$ & $-9.38 \mathrm{~ms}(p=0.15)$ \\
Change in MF-VEP latency at week 32 for opicinumab vs. placebo & $-3.82 \mathrm{~ms}(p=0.50)$ & $-7.55 \mathrm{~ms}(p=0.05)$ \\
RENEW study (Cadavid et al. [3]) & $-3.48 \mathrm{~ms}(p=0.33)$ & $-9.13 \mathrm{~ms}(p=0.01)$ \\
Change in FF-VEP latency at week 24 for opicinumab vs. placebo & $-6.06 \mathrm{~ms}(p=0.07)$ & $54 / 27$ \\
Change in FF-VEP latency at week 32 for opicinumab vs. placebo & $53 / 26$ & \\
Participants with latency recovery at week 24; opicinumab/placebo $(\%)$ &
\end{tabular}

$F F$-VEP full-field visual evoked potential, $I T T$ intent to treat, $M F$-VEP multifocal visual evoked potential, $P P$ per protocol

fellow eye visual pathway following AON treatment with opicinumab represents a protective therapeutic effect from transsynaptic degeneration after AON.

Fourth, it is also possible that the fellow eye amplitude loss represents compensatory functional changes in the visual cortex to adapt to loss of vision in the affected eye resulting from AON. Importantly, the reduced rate of amplitude recovery in the affected eyes and new onset of amplitude loss in the fellow eye in the placebo arm occurred within a similar timeframe, which suggests that the two may be functionally related. These adaptive changes may have been prevented by treatment with opicinumab. The human visual cortex is known to display significant degrees of neuroplasticity [37] that may enable a degree of functional reorganization to compensate for loss of unilateral visual input and, for the brain, a chance to optimize cerebral binocular vision even at the cost of lowering overall amplitude [37-41]. There is some emerging evidence that LINGO-1 signaling pathways are involved in neuroplasticity. One study showed LINGO-1 antagonism increased long-term potentiation (LTP) and prevented the decrease of LTP induced by Nogo-P4; these effects were mediated via Nogo-66 and the ROCK2-Cofilin pathway, which under normal conditions restricts LTP by preventing actin polymerization and maintaining synaptic stability [42]. Furthermore, blocking Nogo-A increased dendritic spine numbers and length in $\mathrm{CA} 3$ pyramidal neurons, while a significant increase in miniature excitatory postsynaptic current amplitude was observed at hippocampal synaptic sites shortly after administration of an anti-NogoA antibody, along with an increase in $\alpha$-amino-3-hydroxy5-methyl-4-isoxazolepropionic acid receptor density that is crucial for plasticity [43]. LINGO-1 has been found to be expressed at distal axonal-synaptic structures [44]. These observations raise the intriguing possibility that opicinumab via LINGO-1 blockade may modulate signaling pathways involved in neuroplasticity.

Limitations of this study include the small sample size. Additionally, as previously reported by Cadavid et al. [3], initiation of opicinumab treatment in RENEW occurred after the majority of retinal thinning had taken place, which limited the therapeutic window for potential treatment benefit on RGC and optic nerve neuroprotection from opicinumab. 


\section{Conclusion}

By generating reliable and informative results in a multicenter international substudy, the feasibility of using MFVEP as a proof-of-concept biomarker for candidate CNS neuroreparative treatment studies in the AON setting has been established. Furthermore, several important observations were made. First, MF-VEP latency changes were highly correlated $(r \geq 0.91)$ with FF-VEP latency changes, corroborating the primary endpoint results in RENEW regarding improvement of VEP latency following AON treatment with opicinumab. Second, the novel finding of progressive fellow eye visual pathway amplitude loss following AON was identified. Third, opicinumab treatment prevented the fellow eye amplitude loss observed following AON and reduced it in the affected eye. If the amplitude loss is due to anterograde transsynaptic degeneration in the visual cortex, this finding raises the possibility that LINGO-1 blockade with opicinumab could function as a neuroprotective therapy for the visual system. If the amplitude loss in the placebo arm is due to plasticity, this indicates that LINGO-1 blockade with opicinumab could be used to modulate neuroplasticity postsynaptically. Both possibilities require further investigation in clinical and experimental settings.

Acknowledgements The authors thank all the investigators and participants in the RENEW MF-VEP substudy. Thanks also are extended to the following for their assistance: the study's contract research organization, Pharmaceutical Product Development, LLC (Wilmington, NC, USA); the staff at NeuroRx Research (Montreal, QC, Canada), who read and interpreted all MRI results; VisionSearch (Sydney, NSW, Australia); and the staff from Duke University (Durham, NC, USA), which was the central reading center. Becky Ayles, Ph.D., from Excel Scientific Solutions wrote the first draft of the manuscript based on input from authors, and Jackie Parker from Excel Scientific Solutions copyedited and styled the manuscript per journal requirements. Biogen reviewed and provided feedback on the paper to the authors. The authors had full editorial control of the paper and provided their final approval of all content. The RENEW MF-VEP investigators are Helmut Butzkueven, Royal Melbourne Hospital, Parkville, VIC, Australia; Ray Garrick, Save Sight Institute, Sydney, NSW, Australia; Ludo Vanopdenbosch, AZ Sint-Jan AV, Brugge, Belgium; Jette Frederiksen, Rigshospitalet Glostrup, Glostrup, Denmark; Orhan Aktas and Philipp Albrecht, Heinrich-Heine-Universität Düsseldorf, Düsseldorf, Germany; Focke Ziemssen, Universitätsklinikum Tübingen, Tübingen, Germany; Tjalf Ziemssen, Universitätsklinikum Carl Gustav Carus, Dresden, Germany; Giancarlo Comi, Ospedale San Raffaele S.r.l., Milan, Italy; Bernardo Sánchez Dalmau, Institut Biomedical Research August Pi Sunyer (IDIBAPS) Center of Neuroimmunology, Barcelona, Spain; Magnus Andersson, Karolinska Universitetssjukhuset Solna, Stockholm, Sweden; Gordon T. Plant, Moorfields Eye Hospital, London, UK; Tim Matthews, Queen Elizabeth Hospital, Birmingham, UK; Graeme Williams, Gartnavel General Hospital, Glasgow, UK.

Author Contributions AK, YC, LX, and DC designed the substudy with input from the other authors and were responsible for the conduct of the substudy. LL, PA, OA, HB, TZ, FZ, and JF collected study data and gave input on analysis. YC and LX analyzed the data and all authors were involved in data interpretation.

\section{Compliance with Ethical Standards}

Funding This study was supported by Biogen (Cambridge, MA, USA). Biogen also provided funding for medical writing support in the development of this paper and for the open access fee.

Conflict of interest Alexander Klistorner has nothing to disclose. Yi Chai was a full-time employee of Biogen during the performance of the clinical trial, data analysis, and initial drafting of the manuscript. Letizia Leocani has received honoraria for consulting and/or speaking activities from Biogen, Merck, Roche, and Teva; research support from Biogen, Merck, and Novartis; and travel support from Almirall, Biogen, Merck, Novartis, and Roche. Philipp Albrecht has received grants from Biogen, the Dr. Robert Pfleger Foundation, Ipsen, Merz, and Novartis; received non-financial support from Bayer HealthCare, Ipsen, Merck, Merz, Novartis, Roche, and Teva; participated in advisory boards for Allergan, Ipsen, and Merz; and received personal fees from Allergan, Bayer HealthCare, Biogen, Ipsen, Novartis, Roche, and Teva, outside of the submitted work. Orhan Aktas has received advisor fees or honoraria from Almirall, Bayer HealthCare, Biogen, MedImmune, Merck, Novartis, and Teva; and research support from Bayer HealthCare, Biogen, Novartis, Roche, and Teva. Helmut Butzkueven has participated in advisory boards for Biogen, Merck, Novartis, and Teva; received consulting fees from Novartis and Oxford Pharmagenesis; lecture fees from Biogen; received payment for development of educational presentations from Biogen, Merck, and Novartis; and research grants from Biogen and Novartis, outside of the submitted work. Tjalf Ziemssen has received consulting fees from Almirall, Bayer HealthCare, Biogen, Genzyme, GlaxoSmithKline, Merck Serono, MSD, Novartis, Sanofi-Aventis, Synthon, and Teva; and research support from Bayer HealthCare, Biogen, Merck Serono, Novartis, SanofiAventis, and Teva. Focke Ziemssen has received consulting fees from Alimera, Allergan, Bayer HealthCare, Boehringer Ingelheim, Novartis, and Roche; and research support from Novartis. Jette Frederiksen has participated in scientific advisory boards for Almirall, Biogen, Genzyme, Merck Serono, Novartis, Sanofi-Aventis, and Teva; received speaker honoraria from Biogen, Merck Serono, Santhera, and Teva; and been an advisor on preclinical development for Takeda, outside of the submitted work. Lei Xu was a full-time employee of Biogen during the performance of the clinical trial, data analysis, and initial drafting of the manuscript. Diego Cadavid was a full-time employee of Biogen during the performance of the clinical trial and a full-time employee of Fulcrum Therapeutics during submission of the manuscript; the work is not related to his employment in Fulcrum Therapeutics. He has patent WO 2016112270 A1: Lingo-1 antagonists and uses for treatment of demyelinating disorders, which is assigned to Biogen.

Ethical approval All investigators obtained approval from their local ethics committees.

Informed consent All participants provided written informed consent and the study was performed in accordance with the International Conference on Harmonisation Good Clinical Practice guidelines and the Declaration of Helsinki.

Open Access This article is distributed under the terms of the Creative Commons Attribution-NonCommercial 4.0 International License (http://creativecommons.org/licenses/by-nc/4.0/), which permits any noncommercial use, distribution, and reproduction in any medium, provided you give appropriate credit to the original author(s) and the source, provide a link to the Creative Commons license, and indicate if changes were made. 


\section{References}

1. Mi S, Pepinsky RB, Cadavid D. Blocking LINGO-1 as a therapy to promote CNS repair: from concept to the clinic. CNS Drugs. 2013;27:493-503.

2. Tran JQ, Rana J, Barkhof F, Melamed I, Gevorkyan H, Wattjes MP, et al. Randomized phase I trials of the safety/tolerability of anti-LINGO-1 monoclonal antibody BIIB033. Neurol Neuroimmunol Neuroinflamm. 2014;1:e18.

3. Cadavid D, Balcer L, Galetta S, Aktas O, Ziemssen T, Vanopdenbosch L, RENEW Study Investigators, et al. Safety and efficacy of opicinumab in acute optic neuritis (RENEW): a randomised, placebo-controlled, phase 2 trial. Lancet Neurol. 2017;16:189-99.

4. Halliday AM, McDonald WI, Mushin J. Delayed visual evoked response in optic neuritis. Lancet. 1972;1:982-5.

5. Hood DC, Odel JG, Winn BJ. The multifocal visual evoked potential. J Neuroophthalmol. 2003;23:279-89.

6. Klistorner A, Arvind H, Garrick R, Graham SL, Paine M, Yiannikas C. Interrelationship of optical coherence tomography and multifocal visual-evoked potentials after optic neuritis. Investig Ophthalmol Vis Sci. 2010;51:2770-7.

7. Creel DJ. Visual evoked potentials. http://webvision.med.utah.edu/ book/electrophysiology/visually-evoked-potentials/. Accessed 5 Jul 2016.

8. Pihl-Jensen G, Schmidt MF, Frederiksen JL. Multifocal visual evoked potentials in optic neuritis and multiple sclerosis: a review. Clin Neurophysiol. 2017;128:1234-45.

9. Klistorner AI, Graham SL, Grigg JR, Billson FA. Multifocal topographic visual evoked potential: improving objective detection of local visual field defects. Investig Ophthalmol Vis Sci. 1998;39:937-50.

10. Hood DC, Odel JG, Zhang X. Tracking the recovery of local optic nerve function after optic neuritis: a multifocal VEP study. Investig Ophthalmol Vis Sci. 2000;41:4032-8.

11. Narayanan D, Cheng H, Tang RA, Frishman LJ. Reproducibility of multifocal visual evoked potential and traditional visual evoked potential in normal and multiple sclerosis eyes. Doc Ophthalmol. 2015;130:31-41.

12. Nebbioso M, Steigerwalt RD, Pecori-Giraldi J, Vingolo EM. Multifocal and pattern-reversal visual evoked potentials vs. automated perimetry frequency-doubling technology matrix in optic neuritis. Indian J Ophthalmol. 2013;61:59-64.

13. Cadavid D, Levin N, Costello F, Rahilly A, Klistorner A. Technical feasibility of implementing multifocal VEP for multicenter clinical trials. Neurology. 2013;80(P02):245.

14. Klistorner A, Graham S, Fraser C, Garrick R, Nguyen T, Paine M, et al. Electrophysiological evidence for heterogeneity of lesions in optic neuritis. Investig Ophthalmol Vis Sci. 2007;48:4549-56.

15. Klistorner A, Arvind H, Garrick R, Yiannikas C, Paine M, Graham SL. Remyelination of optic nerve lesions: spatial and temporal factors. Mult Scler. 2010;16:786-95.

16. Jones SJ, Brusa A. Neurophysiological evidence for long-term repair of MS lesions: implications for axon protection. J Neurol Sci. 2003;206:193-8.

17. International Conference on Harmonisation of Technical Requirements for Registration of Pharmaceuticals for Human Use. ICH harmonized tripartite guideline: guideline for good clinical practice. http://www.ich.org/fileadmin/Public_Web_Site/ICH_Products/ Guidelines/Efficacy/E6/E6_R1_Guideline.pdf. Accessed 5 Jul 2016.

18. World Medical Association. WMA Declaration of Helsinki. Ethical principles for medical research involving human subjects. JAMA. 2013;310:2191-4.

19. Cruz-Herranz A, Balk LJ, Oberwahrenbrock T, Saidha S, MartinezLapiscina EH, Lagreze WA, et al., on behalf of the IMSVISUAL consortium. The APOSTEL recommendations for reporting quantitative optical coherence tomography studies. Neurology. 2016;86:2303-9.

20. Alshowaeir D, Yiannikas C, Garrick R, Parratt J, Barnett MH, Graham SL, et al. Latency of multifocal visual evoked potentials in nonoptic neuritis eyes of multiple sclerosis patients associated with optic radiation lesions. Investig Ophthalmol Vis Sci. 2014;55:3758-64.

21. Klistorner AI, Graham SL. Electroencephalogram-based scaling of multifocal visual evoked potentials: effect on intersubject amplitude variability. Investig Ophthalmol Vis Sci. 2001;42:2145-52.

22. Hubel DH, Wiesel TN. Receptive fields, binocular interaction and functional architecture in the cat's visual cortex. J Physiol. 1962;160:106-54.

23. Klistorner A, Vootakuru N, Wang C, Yiannikas C, Graham SL, Parratt $\mathrm{J}$, et al. Decoding diffusivity in multiple sclerosis: analysis of optic radiation lesional and non-lesional white matter. PLoS One. 2015;10:e122114.

24. Rosenblatt MA, Behrens MM, Zweifach PH, Forman S, Odel JG, Duncan CM, et al. Magnetic resonance imaging of optic tract involvement in multiple sclerosis. Am J Ophthalmol. 1987;104:74-9.

25. Frederiksen JL, Larsson HB, Nordenbo AM, Seedorff HH. Plaques causing hemianopsia or quadrantanopsia in multiple sclerosis identified by MRI and VEP. Acta Ophthalmol (Copenh). 1991;69:169-77.

26. Dasenbrock HH, Smith SA, Ozturk A, Farrell SK, Calabresi PA, Reich DS. Diffusion tensor imaging of the optic tracts in multiple sclerosis: association with retinal thinning and visual disability. $\mathrm{J}$ Neuroimaging. 2011;21:e41-9.

27. Balcer LJ, Miller DH, Reingold SC, Cohen JA. Vision and vision-related outcome measures in multiple sclerosis. Brain. 2015;138:11-27.

28. Gresle MM, Liu Y, Kilpatrick TJ, Kemper D, Wu QZ, Hu B, et al. Blocking LINGO-1 in vivo reduces degeneration and enhances regeneration of the optic nerve. Mult Scler J Exp Transl Clin. 2016;2:2055217316641704.

29. Ranger A, Ray S, Szak S, Dearth A, Allaire N, Murray R, et al. Anti-LINGO-1 has no detectable immunomodulatory effects in preclinical and phase 1 studies. Neurol Neuroimmunol Neuroinflamm. 2017;5:e417.

30. Prins D, Hanekamp S, Cornelissen FW. Structural brain MRI studies in eye diseases: are they clinically relevant? A review of current findings. Acta Ophthalmol. 2016;94:113-21.

31. Balk LJ, Twisk JW, Steenwijk MD, Daams M, Tewarie P, Killestein $\mathrm{J}$, et al. A dam for retrograde axonal degeneration in multiple sclerosis? J Neurol Neurosurg Psychiatry. 2014;85:782-9.

32. Mi S, Hu B, Hahm K, Luo Y, Kam Hui ES, Yuan Q, et al. LINGO-1 antagonist promotes spinal cord remyelination and axonal integrity in MOG-induced experimental autoimmune encephalomyelitis. Nat Med. 2007;13:1228-33.

33. Mi S, Miller RH, Lee X, Scott ML, Shulag-Morskaya S, Shao Z, et al. LINGO-1 negatively regulates myelination by oligodendrocytes. Nat Neurosci. 2005;8:745-51.

34. Lee X, Yang Z, Shao Z, Rosenberg SS, Levesque M, Pepinsky RB, et al. NGF regulates the expression of axonal LINGO-1 to inhibit oligodendrocyte differentiation and myelination. J Neurosci. 2007;27:220-5.

35. Mi S, Miller RH, Tang W, Lee X, Hu B, Wu W, et al. Promotion of central nervous system remyelination by induced differentiation of oligodendrocyte precursor cells. Ann Neurol. 2009;65:304-15.

36. Hu B, So KF, Pepinsky B, Mi S. Blocking LINGO-1 promotes axonal regeneration in the rat optic nerve crush model. Mult Scler. 2013;19:139.

37. Martins Rosa A, Silva MF, Ferreira S, Murta J, Castelo-Branco M. Plasticity in the human visual cortex: an ophthalmology-based perspective. Biomed Res Int. 2013;2013:568354.

38. Werring DJ, Bullmore ET, Toosy AT, Miller DH, Barker GJ, MacManus DG, et al. Recovery from optic neuritis is associated with a 
change in the distribution of cerebral response to visual stimulation: a functional magnetic resonance imaging study. J Neurol Neurosurg Psychiatry. 2000;68:441-9.

39. Hickman SJ, Toosy AT, Jones SJ, Altmann DR, Miszkiel KA, MacManus DG, et al. Serial magnetization transfer imaging in acute optic neuritis. Brain. 2004;127:692-700.

40. Wandell BA, Smirnakis SM. Plasticity and stability of visual field maps in adult primary visual cortex. Nat Rev Neurosci. 2009;10:873-84.

41. Wong AM. New concepts concerning the neural mechanisms of amblyopia and their clinical implications. Can J Ophthalmol. 2012;47:399-409.
42. Iobbi C, Korte M, Zagrebelsky M. Nogo-66 restricts synaptic strengthening via Lingo1 and the ROCK2-Cofilin pathway to control actin dynamics. Cereb Cortex. 2017;27:2779-92.

43. Kellner Y, Fricke S, Kramer S, Iobbi C, Wierenga CJ, Schwab ME, et al. Nogo-A controls structural plasticity at dendritic spines by rapidly modulating actin dynamics. Hippocampus. 2016;26:816-31.

44. Kuo SH, Tang G, Louis ED, Ma K, Babji R, Balatbat M, et al. Lingo-1 expression is increased in essential tremor cerebellum and is present in the basket cell pinceau. Acta Neuropathol. 2013;125:879-89. 